\title{
Correction to: Histological classification of breast tumors in the General Rules for Clinical and Pathological Recording of Breast Cancer (18th edition)
}

\author{
Hitoshi Tsuda ${ }^{1}$ (1) $\cdot$ Koichiro Tsugawa ${ }^{2} \cdot$ Futoshi Akiyama $^{3} \cdot$ Rie Horii $^{4} \cdot$ Masafumi Kurosumi $^{5} \cdot$ Takuya Moriya $^{6}$. \\ Toshimi Takano ${ }^{7} \cdot$ Hiroyuki Takei $^{8} \cdot$ Takahiro Nakayama $^{9} \cdot$ Yumi Miyagi $^{10}$. Chikako Yamauchi ${ }^{11}$. \\ Toshinari Yamashita $^{12} \cdot$ Kenjiro Aogi $^{13} \cdot$ Hirofumi Mukai $^{14} \cdot$ Tomoharu Sugie $^{15} \cdot$ Hiroji Iwata $^{16} \cdot$ Shinobu Masuda $^{17}$
}

Published online: 19 May 2020

(c) The Japanese Breast Cancer Society 2020

\section{Correction to: Breast Cancer (2020) 27:309-321 https://doi.org/10.1007/s12282-020-01074-3}

In the original publication of the article, the author group and acknowledgements were published incorrectly. The correct author group and acknowledgements are given in this correction.

The original article can be found online at https://doi.org/10.1007/ s12282-020-01074-3.

Hitoshi Tsuda

htsuda@ndmc.ac.jp

1 Department of Basic Pathology, National Defense Medical College, 3-2 Namiki, Tokorozawa, Saitama 359-8513, Japan

2 Division of Breast and Endocrine Surgery, Department of Surgery, St. Marianna University School of Medicine, 2-16-1Miyamae-ku, SugaoKawasaki, Kanagawa 216-8511, Japan

3 Division of Pathology, Clinicopathology Center, The Cancer Institute of Japanese Foundation for Cancer Research, 3-8-31 Ariake, Koto-ku, Tokyo 135-8550, Japan

4 Department of Pathology, Saitama Cancer Center, 780 Komuro, Ina-machi, Kita-adachi-gun, Saitama 362-0806, Japan

5 Division of Pathology, Breast Center, Kameda Medical Center, Tokyo Square Garden, 3-1-1 Kyobashi, Chuo-ku, Tokyo 104-0031, Japan

6 Department of Pathology, Kawasaki Medical School, 577 Matsushima, Kurashiki, Okayama 701-0192, Japan

7 Department of Medical Oncology, Toranomon Hospital, 2-2-2 Toranomon, Minato-ku, Tokyo 105-8470, Japan

8 Graduate School and Department of Breast Surgery and Oncology, Nippon Medical School, 1-1-5 Sendagi, Bunkyo-ku, Tokyo 113-8603, Japan
Acknowledgements The authors greatly thanks to Dr. Shu Ichihara, Department of Pathology, National Hospital Organization Nagoya Medical Center, Nagoya, Japan, for intensive and instructing discussion as a working group member for histological classification.

Publisher's Note Springer Nature remains neutral with regard to jurisdictional claims in published maps and institutional affiliations.

9 Department of Breast and Endocrine Surgery, Osaka International Cancer Institute, 3-1-69, Otemae, Chuo-ku, Osaka 541-8567, Japan

10 Department of Breast Surgery, Breast Oncology Center, Cancer Institute Hospital, Japanese Foundation for Cancer Research, 3-8-31 Ariake, Koto-ku, Tokyo 135-8550, Japan

11 Department of Radiation Oncology, Shiga General Hospital, 5-4-30 Moriyama, Moriyama-shi, Shiga 524-8524, Japan

12 Department of Breast and Endocrine Surgery, Kanagawa Cancer Center, 2-3-2 Nakao, Asahi-ku, Yokohama, Kanagawa 241-0815, Japan

13 Division of Clinical Research Promotion, National Hospital Organization Shikoku Cancer Center, 160 Kou, Minami-umemoto-machi, Matsuyama, Ehime 791-0280, Japan

14 Department of Breast and Medical Oncology, National Cancer Center Hospital East, 6-5-1 Kashiwanoha, KashiwaChiba 277-8577, Japan

15 Breast Surgery, Kansai Medical University Hospital, 2-3-1 Shinmachi, Hirakata, Osaka 573-1191, Japan

16 Department of Breast Oncology, Aichi Cancer Center, 1-1 Kanokoden, Chikusa-ku, Nagoya, Aichi 464-8681, Japan

17 Division of Oncologic Pathology, Department of Pathology and Microbiology, Nihon University School of Medicine, 30-1 Oyaguchi Kami-cho, Itabashi-ku, Tokyo 173-8610, Japan 